\title{
A new class of exact solutions of the Navier-Stokes equations for swirling flows in porous and rotating pipes
}

\author{
A. Fatsis ${ }^{1}$, J. Statharas ${ }^{2}$, A. Panoutsopoulou ${ }^{3} \&$ N. Vlachakis ${ }^{1}$ \\ ${ }^{I}$ Technological University of Chalkis, \\ Department of Mechanical Engineering, Greece \\ ${ }^{2}$ Technological University of Chalkis, \\ Department of Aeronautical Engineering, Greece \\ ${ }^{3}$ Hellenic Defence Systems, Greece
}

\begin{abstract}
Flow field analysis through porous boundaries is of great importance, both in engineering and bio-physical fields, such as transpiration cooling, soil mechanics, food preservation, blood flow and artificial dialysis. A new family of exact solution of the Navier-Stokes equations for unsteady laminar flow inside rotating systems of porous walls is presented in this study. The analytical solution of the Navier-Stokes equations is based on the use of the Bessel functions of the first kind. To resolve these equations analytically, it is assumed that the effect of the body force by mass transfer phenomena is the 'porosity' of the porous boundary in which the fluid moves.

In the present study the effect of porous boundaries on unsteady viscous flow is examined for two different cases. The first one examines the flow between two rotated porous cylinders and the second one discusses the swirl flow in a rotated porous pipe. The results obtained reveal the predominant features of the unsteady flows examined. The developed solutions are of general application and can be applied to any swirling flow in porous axisymmetric rotating geometries.
\end{abstract}

Keywords: exact solution, Navier-Stokes, porous, viscous flow, unsteady flow, laminar flow, swirl flow, Bessel functions. 


\section{Introduction}

In the previous years, problems of fluid flow through porous ducts have aroused the interest of Engineers and Mathematicians; the problems have been studied for their possible applications in cases of transpiration cooling, gaseous diffusion and drinking water treatment, as well as biomedical engineering. The cases where an exact solution for the Navier-Stokes equations can be obtained are of particular importance in order to describe the fluid motion of viscous flows. However, since the Navier-Stokes equations are non-linear, there cannot be a general method to solve analytically the full equations. Exact solutions on the other hand are very important for many reasons. They provide a reference solution to verify the accuracies of many approximate methods, such as numerical and/or empirical. Although, nowadays, computer techniques make the complete integration of the Navier-Stokes equations feasible, the accuracy of numerical results can be established only by comparison with an exact solution [1]. The Navier-Stokes equations were extensively studied in the literature. Exact solutions already known are one-dimensional or parallel shear flows, rectilinear motion flows, or duct flows [1-3]. The flow of fluids over boundaries of porous materials has many applications in practice, such as boundary layer control and transpiration processes. Exact solutions are generally easy to find when suction or injection is applied to a fluid flow. In the case of flows through porous media, a simple solution of the Navier-Stokes equations can be obtained for the flow over a porous plane boundary at which there is a uniform suction velocity [4]. Moreover, fully developed laminar flow through porous channel with a porous pipe for low Reynolds numbers was investigated in [5] and the flow in a duct of rectangular cross-section in [6]. This problem was extended in [7] to high Reynolds numbers. The exact solution of the Navier-Stokes equations for the case of steady laminar flow between two porous coaxial cylinders with different permeability was obtained using the perturbation technique [8]. The cylinders were assumed to rotate with different angular velocities and the fluid between them was flowing with a constant axial pressure gradient. A mathematical model for particle motion in viscous flow between two rotating porous cylinders was also presented [9]. In that paper, a steady flow of a mixture of fluid and particles was assumed. The mass fraction of particles in the flow was small, so the perturbations of the mean liquid flow due to the presence of particles were negligible. An analytical approximate solution for decaying laminar swirling flows within a narrow annulus between two concentric cylinders was also obtained. It was found that the swirl velocity exhibits a Hagen-Poiseuille flow profile decaying downstream [10]. An exact solution of the Navier-Stokes equation was obtained in [11] for the laminar incompressible flow in a uniformly porous pipe with suction and injection. In this study the velocity field was expressed in a series form in terms of the modified Bessel function of the first kind of order $n$. For large values of the non-dimensional time, the unsteady flow solution approaches its asymptotic value of the steady state problem. Laminar flow over pipes with injection and suction through the 
porous wall was studied by means of analytic solutions for the case of low Reynolds numbers [12].

In the present study the full unsteady three-dimensional Navier-Stokes equations are considered for the case of incompressible porous flow. An exact solution is obtained by employing the Bessel functions for the case of threedimensional unsteady flow between rotated porous cylinders and for the case of unsteady swirl flow in rotated porous pipes.

\section{Mathematical and physical modelling}

Assuming for the first case study the flow of a Newtonian fluid through an annulus formed between two rotating cylinders, figure 1a, and, for the second case study the flow within a rotating cylindrical pipe, figure $1 \mathrm{~b}$, the basic equations are the mass conservation equation and the equations of motion (Navier-Stokes), in a cylindrical system of coordinates $(r, \theta, z)$ where the $z$-axis lies along the centre of the pipe, $r$ is the radial distance and $\theta$ is the peripheral angle.

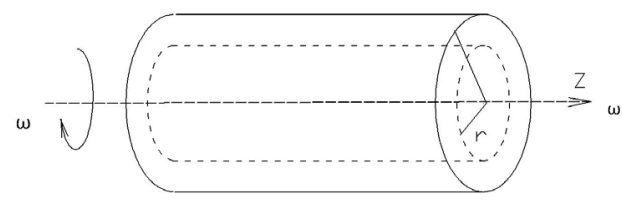

(a)

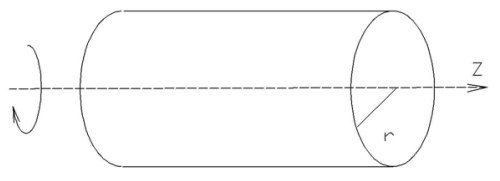

(b)

Figure 1: (a) Flow between two rotating porous cylinders; (b) flow within a rotating porous pipe.

\subsection{Governing equations}

Considering that the flow modelling describes the motion of a homogeneous Newtonian fluid the incompressible Navier-Stokes equations are the governing equations, while the following simplified assumptions are made:

a) the rotating cylinders or the rotating pipe are considered of finite length

b) the permeable wall boundary is treated as a 'fluid medium'.

c) the gravitational forces due to the fluid weight are negligible.

The continuity equation is:

$$
\frac{u_{r}}{r}+\frac{\partial u_{r}}{\partial r}+\frac{\partial u_{z}}{\partial z}+\frac{1}{r} \cdot \frac{\partial u_{\theta}}{\partial \theta}=0
$$

The system of the Navier-Stokes equations can be written:

$$
\frac{\partial u_{r}}{\partial t}+u_{r} \frac{\partial u_{r}}{\partial r}-\frac{u_{\theta}^{2}}{r}+u_{z} \frac{\partial u_{r}}{\partial z}=-\frac{1}{\rho} \cdot \frac{\partial p}{\partial r}+\omega^{2} r+2 \omega u_{\theta}+
$$




$$
\begin{array}{r}
\frac{\mu}{\rho}\left[\frac{\partial^{2} u_{r}}{\partial r^{2}}+\frac{1}{r} \cdot \frac{\partial u_{r}}{\partial r}-\frac{u_{r}}{r^{2}}+\frac{\partial^{2} u_{r}}{\partial z^{2}}\right] \\
\frac{\partial u_{\theta}}{\partial t}+u_{r} \frac{\partial u_{\theta}}{\partial r}+\frac{u_{r} \cdot u_{\theta}}{r}+u_{z} \frac{\partial u_{\theta}}{\partial z}=-\frac{1}{\rho} \cdot \frac{1}{r} \cdot \frac{\partial P}{\partial r}-2 \omega u_{r}+ \\
\frac{\mu}{\rho}\left[\frac{\partial u_{\theta}^{2}}{\partial r^{2}}+\frac{1}{r} \cdot \frac{\partial u_{\theta}}{\partial r}-\frac{u_{\theta}}{r^{2}}+\frac{\partial^{2} u_{\theta}}{\partial z^{2}}\right] \\
\frac{\partial u_{z}}{\partial t}+u_{r} \frac{\partial u_{z}}{\partial r}+u_{z} \frac{\partial u_{z}}{\partial z}=-\frac{1}{\rho} \cdot \frac{\partial p}{\partial z}+\frac{\mu}{\rho}\left[\frac{\partial^{2} u_{z}}{\partial r^{2}}+\frac{1}{r} \cdot \frac{\partial u_{z}}{\partial r}+\frac{\partial^{2} u_{z}}{\partial z^{2}}\right]
\end{array}
$$

\section{Solution methodology}

\subsection{Unsteady flow between two coaxial porous rotating cylinders}

An incompressible fluid of dynamic viscosity $\mu$ and density $\rho$ is considered between two rotating cylinders of length $L$. The inner cylinder can rotate with peripheral velocity $\omega \cdot R_{i}$ and the outer can rotate with peripheral velocity $\omega \cdot R_{o}$. At time level $t=t_{0}$ the fluid enters the cylinders gap uniformly at $z^{*}=0$ and exits at $z^{*}=L$ or at non-dimensional axial distance $z=1$.

The following boundary conditions are satisfied:

$$
\begin{array}{llll}
\text { For } r=R_{i} & \left.u_{r}\right|_{r=R_{i}}=u_{r i} & \left.u_{\theta}\right|_{r=R_{i}}=u_{\theta i}=\omega \cdot R_{i} & \left.u_{z}\right|_{r=R_{i}}=1 \\
\text { For } r=R_{o} & \left.u_{r}\right|_{r=R_{o}}=u_{r o} & \left.u_{\theta}\right|_{r=R_{o}}=u_{\theta o}=\omega \cdot R_{o} & \left.u_{z}\right|_{r=R_{o}}=0
\end{array}
$$

For the test case selected, the value of the axial velocity was set equal to zero at the outer radius. Resolving the system of equations (1) to (4), it was found that the axial velocity $u_{z}$, the radial velocity $u_{r}$ and the tangential velocity $u_{\theta}$, can be expressed in terms of the functions:

$$
\begin{gathered}
u_{z}=J_{0}(r b) e^{-b z} e^{k t}+A \cdot\left(1-r^{2}\right) \\
u_{r}=J_{1}(r b) e^{-b z} e^{k t}+\frac{C}{r} \\
u_{\theta}=\frac{D}{r} e^{k t}
\end{gathered}
$$


where $A, C, D$ are integration constants and $J_{0}(r b)$ and $J_{1}(r b)$ are the Bessel functions of the First kind given in detail in [13].

The constant $D$ is defined as $D=\omega_{1}-\omega_{2}$, so it covers the case of counterrotating cylinders or cylinders rotated in the counter-clockwise direction.

The static pressure field is then calculated analytically as:

$$
p(r, z, \theta, t)=\frac{k}{b} J_{0} e^{-b z} e^{k t}-\frac{1}{2}\left(J_{1}^{2}+J_{0}^{2}\right) e^{-2 b z} e^{2 k t}-\frac{C J_{1}}{r} e^{-b z} e^{k t}-
$$

$\frac{2 A}{b} J_{1} r e^{-b z} e^{k t}+2 A C z-\frac{4}{\operatorname{Re}} z-A J_{0} e^{-b z} e^{k t}+A J_{0} r^{2} e^{-b z} e^{k t}-k D e^{k t} \theta(6)$

where the Reynolds number is defined as: $\operatorname{Re}=\frac{\rho \cdot U \cdot L}{\mu}$

The proposed solution was validated for the case of the laminar fully developed swirling flow in the annulus between two coaxial cylinders. For this case, one can find in the literature numerical solutions as well as analytical ones [10]. Figure 2 presents the comparison between the axial velocity obtained by the present analytical method (solid line) and the analytical solution obtained in [10] (dashed line). The comparison is considered satisfactory to validate the present method, since the maximum difference between these results does not exceed $5 \%$.

The solution of the Navier-Stokes equations defined by equations (5) and (6) does satisfy the continuity equation (1) and momentum equations (2) to (4).

Figure 3 shows the radial velocity distribution for three different time levels, namely for $t=0,1,2$ along the radius. It was assumed that the inner nondimensional (by the cylinders length $L$ ) radius is $R_{i}=0.1$, while the outer

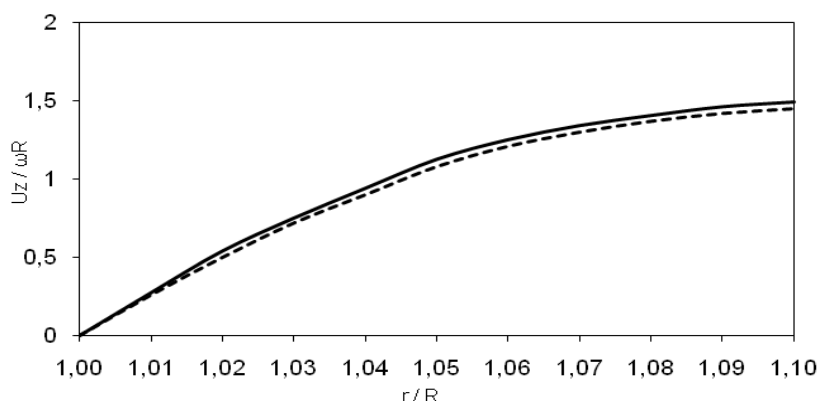

Figure 2: Distribution of the axial velocity for the case of swirling flow between two cylinders. (solid line: present results, dashed line: results from [10]). 


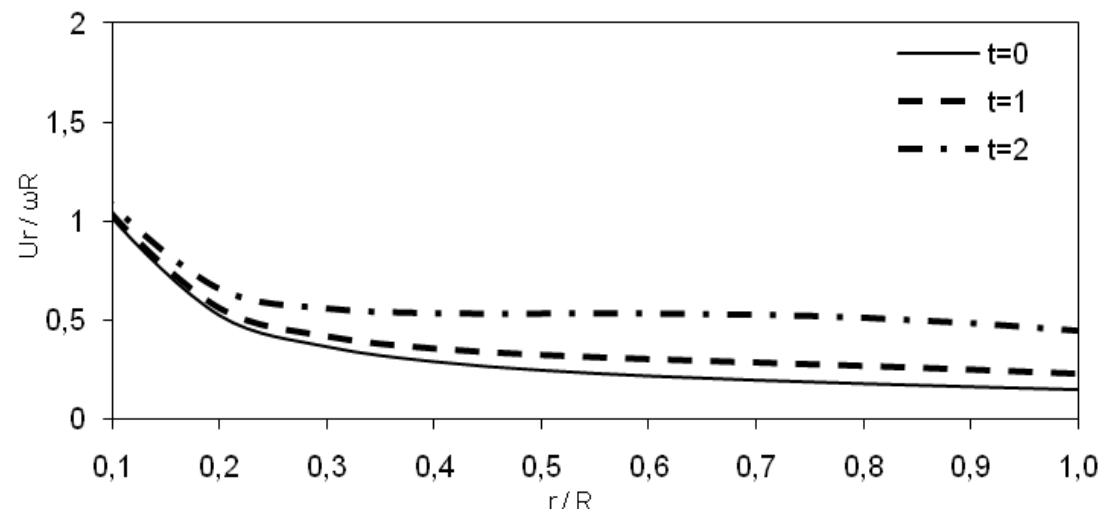

Figure 3: Distribution of the radial velocity in terms of radius for different time levels.

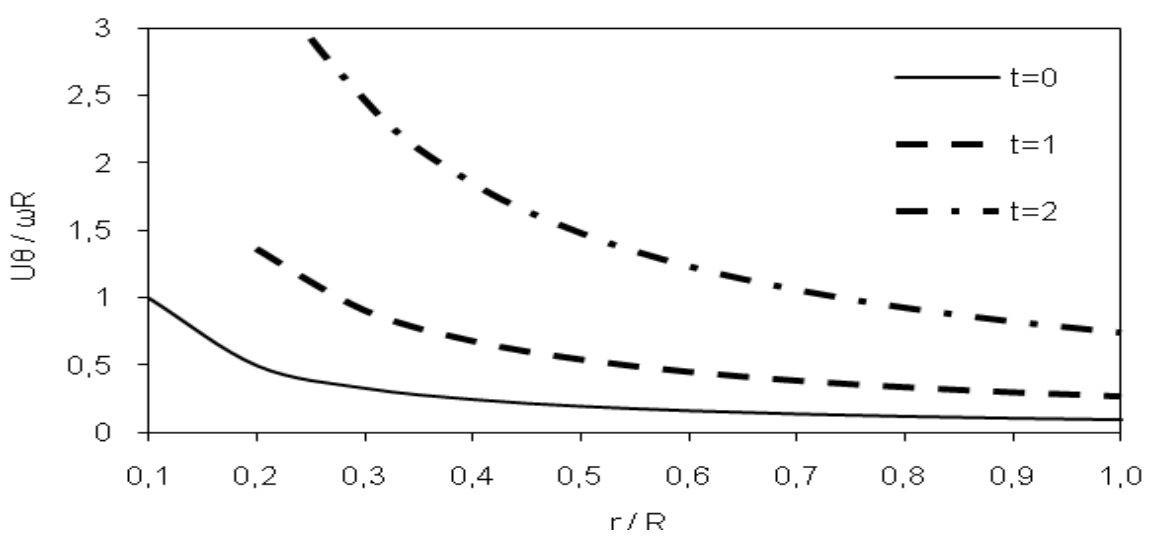

Figure 4: Distribution of the tangential velocity in terms of radius for different time levels.

non-dimensional radius is $R_{o}=0.8$ for specific values of the constants $A, C, D, k$. A decaying behaviour is observed along the radial gap.

The tangential velocity distribution shows in figure 4 a reduction from the inner to the outer cylinder for $t=0,1,2$. As the time level increases more fluid is moving tangentially, thus tangential velocity values increase.

The axial velocity has a decaying distribution from the inner to the outer radius, figure 5 .

Figure 6 shows the radial velocity distribution for a given time level for different non-dimensional axial positions, namely for $z=0,0.3,0.8$. 


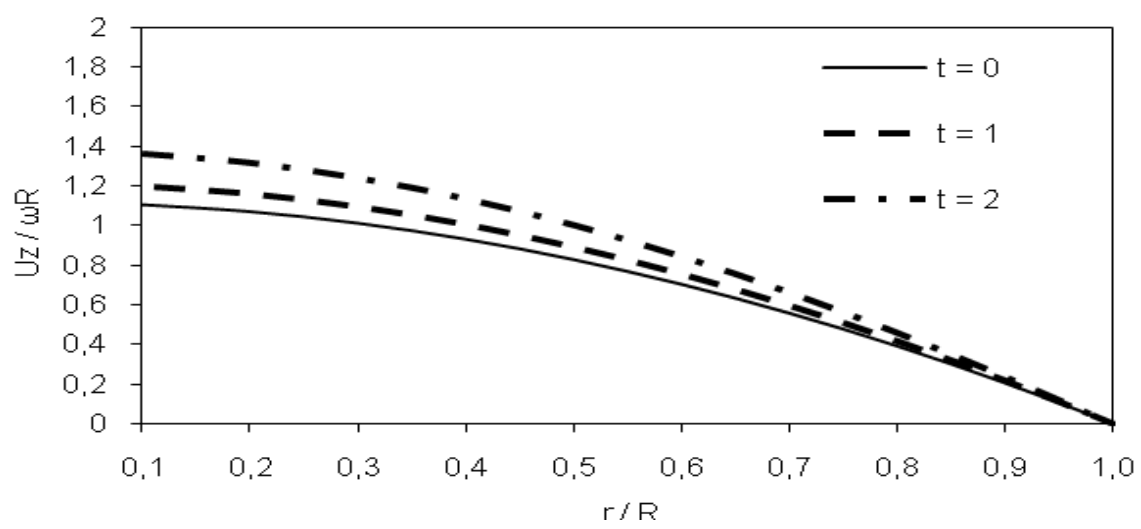

Figure 5: Distribution of the axial velocity in terms of radius for different time levels.

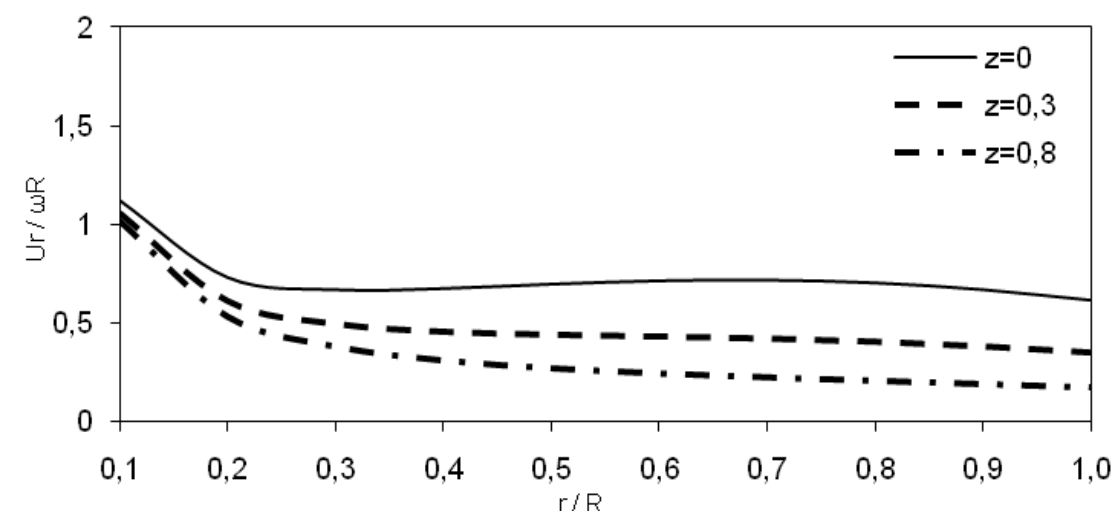

Figure 6: Distribution of the radial velocity in terms of radius for different axial positions.

The radial velocity decreases from the inlet to the outlet. The distribution of the tangential velocity found to satisfy the system of equations of motion is not a function of the axial distance, $z$ according to equation $(5 \mathrm{c})$. So at any axial position, the tangential position has a constant radial distribution at given time levels.

Figure 7 presents the axial velocity distribution for a given time level for different axial positions $z=0,0.3,0.8$. The axial velocity shows an increase along the gap of the cylinders from the inlet to the outlet. 


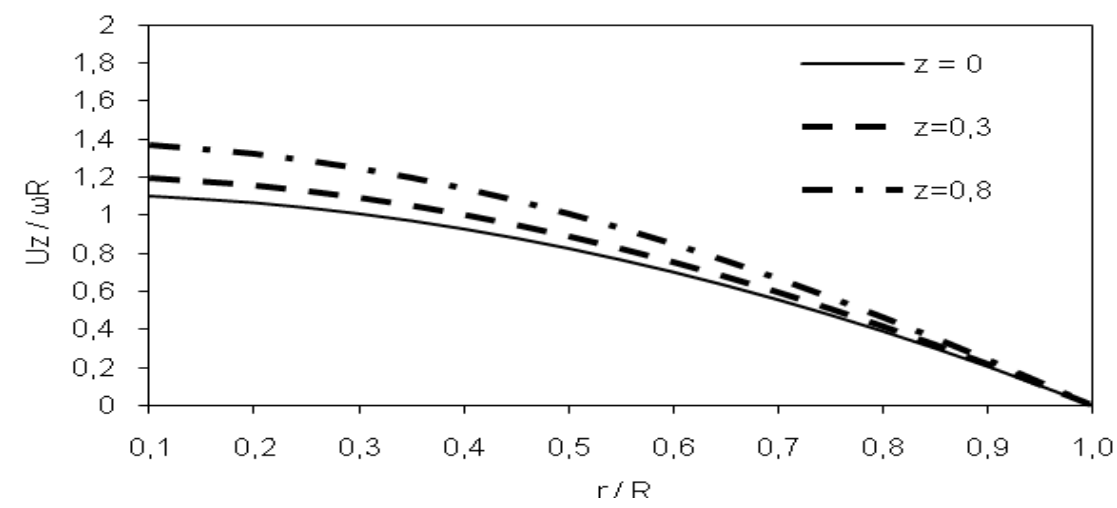

Figure 7: Distribution of the axial velocity in terms of radius for different axial positions.

\subsection{Unsteady swirling flow in a rotated porous pipe}

An incompressible fluid of dynamic viscosity $\mu$ and density $\rho$ is considered within a rotating pipe of length $L$. The inner pipe can rotate along its axis with peripheral velocity $\omega \cdot R$. At time level $t=t_{0}$ the fluid enters the pipe uniformly at $z^{*}=0$ and exits at $z^{*}=L$ or at non-dimensional axial distance $z=1$.

The following boundary conditions are satisfied:

$\begin{array}{llll}\text { For } r=0 & \left.u_{r}\right|_{r=R_{i}}=0 & \left.u_{\theta}\right|_{r=R_{i}}=0 & \left.u_{z}\right|_{r=0}=1 \\ \text { For } r=R_{o} & \left.u_{r}\right|_{r=R_{o}}=u_{r o} & \left.u_{\theta}\right|_{r=R_{o}}=u_{\theta o}=\omega \cdot R_{o} & \left.u_{z}\right|_{r=R_{o}}=0\end{array}$

For the test case selected, the value of the axial velocity is zero at the outer radius.

The axial velocity $u_{z}$, the radial velocity $u_{r}$ and the tangential velocity $u_{\theta}$, can expressed in terms of the functions:

$$
\begin{gathered}
u_{z}=J_{0}(r b) e^{-b z} e^{k t}+A \cdot\left(1-r^{2}\right) \\
u_{r}=J_{1}(r b) e^{-b z} e^{k t}+\frac{B}{r} \\
u_{\theta}=C \cdot r
\end{gathered}
$$

where $J_{0}(r b)$ and $J_{1}(r b)$ are the Bessel functions of the First kind and $A, B, C$ are integration constants. 
The static pressure field is then calculated analytically as:

$$
\begin{gathered}
p(r, z, \theta, t)=\frac{k}{b} J_{0} e^{-b z} e^{k t}-\frac{1}{2}\left(J_{1}^{2}+J_{0}^{2}\right) e^{-2 b z} e^{2 k t}+\frac{B J_{1}}{r} e^{-b z} e^{k t}- \\
\frac{2 A}{b} J_{1} r e^{-b z} e^{k t}-A J_{0} e^{-b z} e^{k t}+A J_{0} r^{2} e^{-b z} e^{k t}+2 A B z+A z
\end{gathered}
$$

The solution of the Navier-Stokes equations defined by equations (7) and (8) does satisfy the continuity equation (1) and momentum equations (2) to (4).

Figure 8 shows the radial velocity distribution for three different time levels, namely for $t=0,1,2$ along the radius. A decaying behaviour is observed in this figure. The tangential velocity distribution found in equation (11c) is independent of the time variable $t$.

The axial velocity has a decaying distribution towards the outer radius, figure 9 .

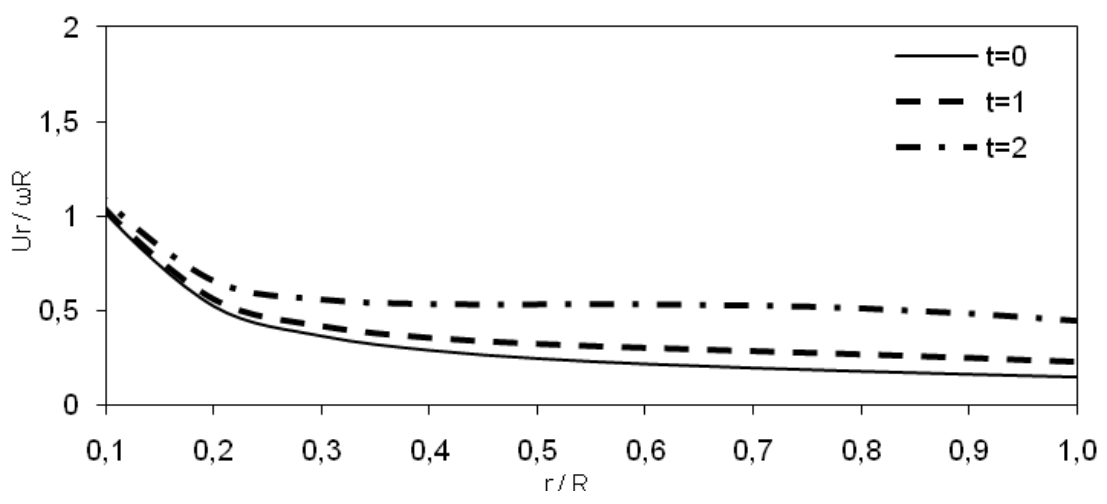

Figure 8: Distribution of the radial velocity in terms of radius for different time levels.

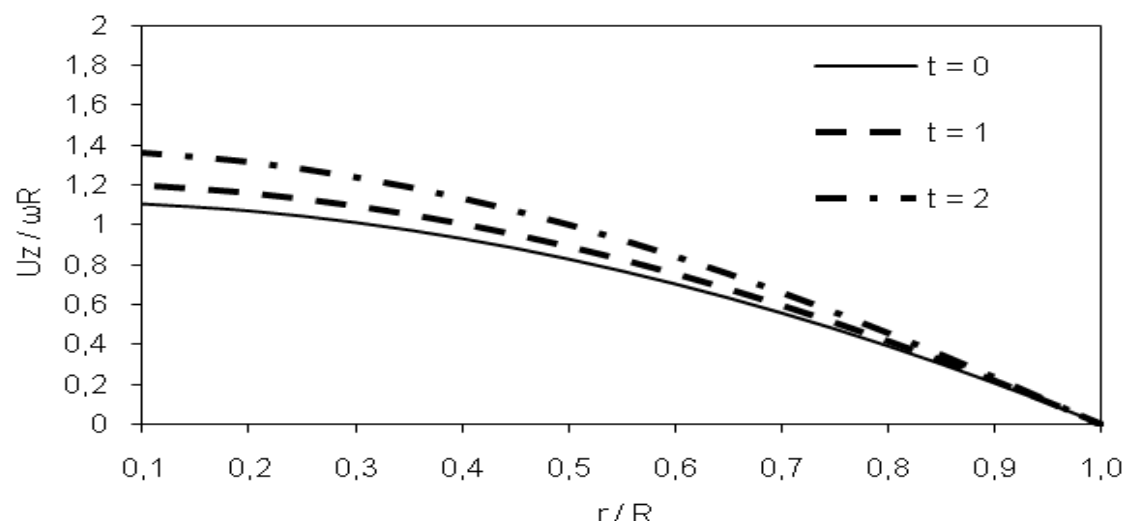

Figure 9: Distribution of the axial velocity in terms of radius for different time levels. 


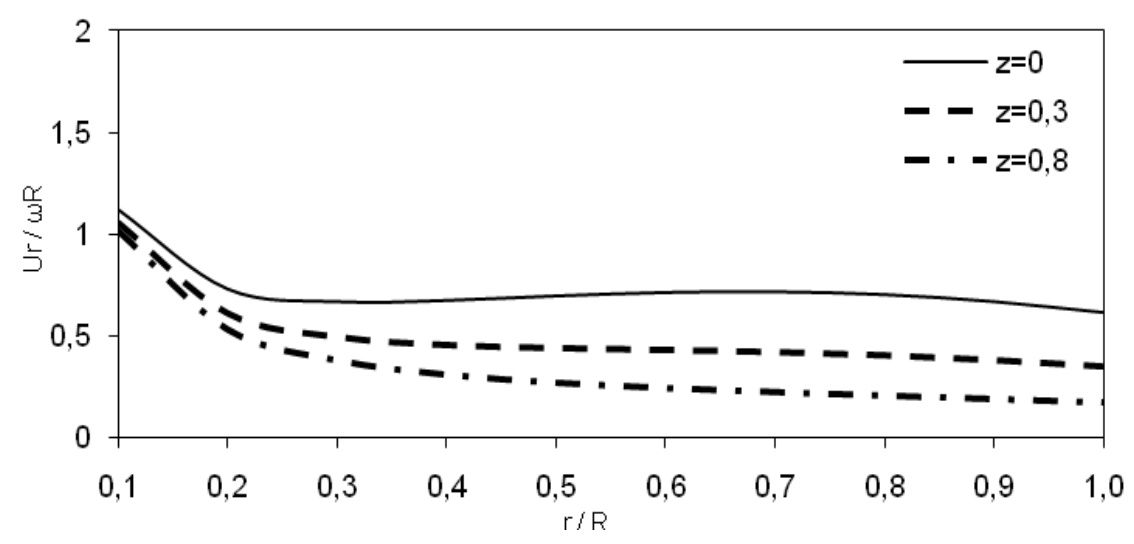

Figure 10: Distribution of the radial velocity in terms of radius for different axial positions.

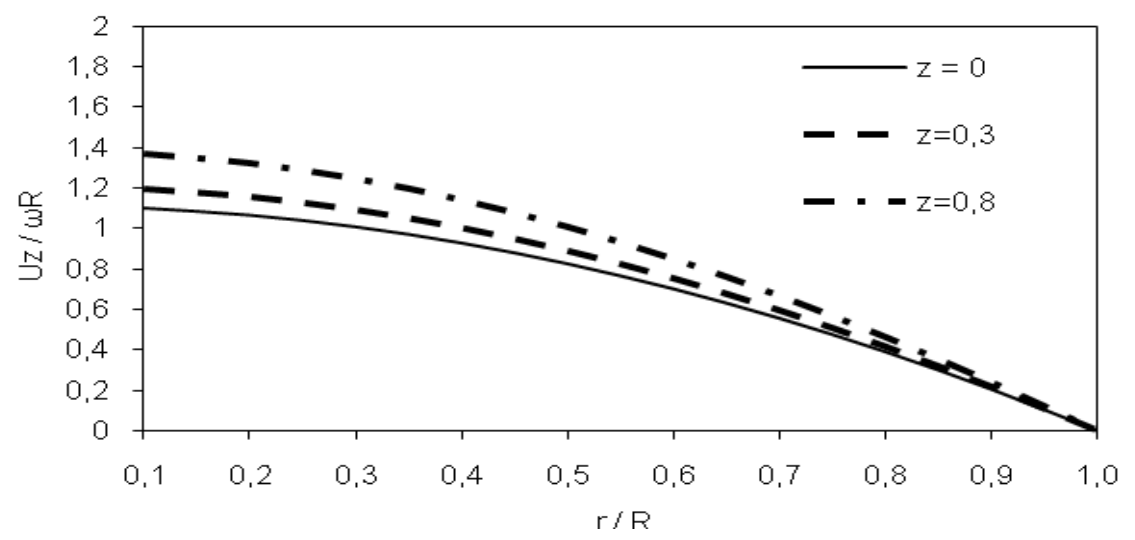

Figure 11: Distribution of the axial velocity in terms of radius for different axial positions.

Figure 10 shows the radial velocity distribution for a given time level for different non-dimensional axial positions, namely for $z=0,0.3,0.8$.

The distribution of the tangential velocity found to satisfy the system of equations of motion is not a function of the axial distance, $z$. So at any axial position, the tangential position has a constant radial distribution at given time levels.

Figure 11 presents the axial velocity distribution for a given time level for different axial positions $z=0,0.3,0.8$. The axial velocity shows an increase along the gap of the cylinders from the inlet to the outlet. 


\section{Conclusions}

In this article, an original work presenting exact solutions of the Navier-Stokes equations in the presence of porous boundaries of axisymmetric rotating geometries is proposed. Such flows have significant industrial applications including filtration and particle separation.

Two cases were examined. The first one is the unsteady flow between two rotating porous cylinders and the second one is the unsteady flow inside rotating porous pipes. In both cases, the Bessel functions of the first kind were used to compute the axial and radial components of the flow velocities, while the tangential flow velocity was found to depend only on the radius. For both cases, the velocity and pressure fields were found by means of analytical methods to satisfy the Navier-Stokes equations for laminar, incompressible unsteady flows.

For the case of the unsteady flow inside two rotating cylinders, it was found that the maximum of the axial velocity shifts towards the inner cylinder. The axial and radial velocity components are independent of the rates of rotation of cylinders. The tangential flow velocity having the form of "free vortex" type of flow was found to satisfy the equations of motion.

For the case of the swirl flow inside rotating pipes, it was found that the maximum of the axial velocity is at the centre of the pipe and decays towards the porous boundary at the maximum radius. Variations were observed also for the radial velocity component which also has a maximum close to the centre of the pipe. The linear variation of the tangential velocity having the form of "forced vortex" type of flow along the radius was found to satisfy the equations of motion.

\section{References}

[1] Turkyilmazoglu, M, Exact solutions for the incompressible viscous fluid of a porous rotating disk, International Journal of Non-Linear Mechanics, 44 (2009), pp. 352-357.

[2] Batchelor, G.K., An Introduction to Fluid Dynamics, Cambridge University Press, Cambridge, 1967.

[3] Polyanin A.D., Exact solution to the Navier-Stokes equations with generalized separation of variables, Dokt. Phys. 46 (2001), pp. 726-731.

[4] Sherman, R.S., Viscous Flow, McGraw-Hill Inc., New York, 1990.

[5] Berman, A.S., Laminar flow in channels with porous walls, J. Appl. Phys., 24 (1953), pp. 1232-1235.

[6] Jain, R.K. Metha, K.N., Laminar hydrodynamic flow in a rectangular channel with porous walls, Proc. Nat. Inst. Sci. India, 28 (1962), pp. 846856.

[7] Terril R.M., Laminar flow in a uniformly porous channel, The Aeronautical Quarterly 15, 1964, pp.299.

[8] Gupta, M.C., Goyal, M.C., Viscous incompressible steady laminar flow between two porous coaxial rotating circular cylinders with different 
permeability, Indian Journal of pure applied Mathematics, Vol. 3, No. 3, pp.402-425.

[9] Gumerov, N. A., Dureiswami, R., Modeling of particle motion in viscous swirl flow between two porous cylinders, ASME Paper FEDSM98-5110 in the Proceedings of 1998 ASME Fluids Engineering Division Summer Meeting, June 21-25, 1998.

[10] Jawarneh, A.M., Vatistas, G.H., Ababneh, A., Analytical approximate solution for decaying laminar swirling flows within a narrow annulus, Jordan Journal of Mechanical and Industrial Engineering, 2 (2008), pp. 101-109.

[11] Erdogan, M.E., Imrak, C.E., On the flow in a uniformly porous pipe, International Journal on Non-Linear Mechanics, 43 (2008), pp. 292-301.

[12] Moussy, Y., Snider, A.D., Laminar flow over pipes with injection and suction through the porous wall at low Reynolds number, Journal of Membrane Science, 327 (2009), pp. 104-107.

[13] N. Vlachakis, A. Fatsis, A. Panoutsopoulou, E. Kioussis M. Kouskouti V. Vlachakis, An exact solution of the Navier-Stokes equations for swirl flow models through porous pipes, Proceedings of the $6^{\text {th }}$ International Conference on Advances in Fluid Mechanics, Wessex Institute of Technology, (2006), pp. 583-591. 